\title{
EVALUACIÓN Y AFRONTAMIENTO DE LOS AJETREOS DIARIOS: RELACIÓN CON OPTIMISMO, PERFECCIONISMO, ALEXITIMIA Y AFECTO POSITIVO Y NEGATIVO
}

\author{
JESÚS ZORROZA, JOSÉ SORIANO y JOSÉ SÁNCHEZ-CÁNOVAS \\ Universidad de Valencia
}

(Recibido el 7 de septiembre de 1998)

\begin{abstract}
El propósito del trabajo es comprobar si los contenidos de la evaluación y los modos de afrontamiento que se llevan a cabo ante los ajetreos diarios se pueden explicar a partir de 4 dimensiones de personalidad: afectividad, optimismo, perfeccionismo y alexitimia. Asimismo, se pretende averiguar si los modos concretos de afrontamiento están más influidos por la evaluación concreta que el sujeto realiza sobre la situación, que por estos rasgos de personalidad. Los resultados indican que estas dimensiones, consideradas como factores relativamente estables de la persona, poseen una repercusión clara y poderosa tanto en la valoración que se hace de los acontecimientos, como en la selección de las conductas específicas de afrontamiento. Además, la búsqueda del apoyo social depende sobre todo de estas dimensiones de personalidad y, en mucha menor medida, de los contenidos de evaluación primaria y secundaria de la situación. La evitación está marcadamente condicionada por esas variables de personalidad y sólo de manera moderada por la evaluación. Por último, la utilización de estrategias de solución de problemas, depende por igual de ambos tipos de constructos.
\end{abstract}

Palabras clave: Estrés, afrontamiento, evaluación, afectividad, alexitimia, optimismo, perfeccionismo.

Appraisal and ways of coping with daily hassles: relationships with optimism, perfectionism, alexithymia, and positive and negative affect

The aim of the present work has been to asses to what extent the contents of the appraisal and the ways of coping with the hassles could be explained on the basis of four personality dimensions: positive and negative affect, optimism, perfectionism and alexithymia. Moreover, it has been tested to what extent the explicit ways of coping are influenced by a specific appraisal or, by contrast, are the personality dimensions the main source of influence. Our results showed that the personality dimensions had a powerful and reliable effect on the appraisal of the events and also on the specific ways of coping. The search for social support basically relies on the personality dimensions but slightly on the contents of the primary and secondary appraisal of the situation. The avoidance is highly influenced by these personality traits and just moderately by the appraisal of the events. Finally, the use of problem solving strategies depends on both types of variables.

Key words: Stress, coping, appraisal, affect, alexithymia, optimism, perfectionism.

\section{INTRODUCCIÓN}

En la conceptuación del afrontamiento del estrés han existido dos posturas que son el reflejo de dos planteamientos teóricos genéricos dentro del estudio del compor-

Correspondencia: Jesús Zorroza. Departamento de Personalidad Evaluación y Tratamientos Psicológicos. Facultad de Psicología. Universidad de Valencia. tamiento humano: las aproximaciones disposicionales y las contextuales. Las disposicionales asumen que factores relativamente estables de la persona poseen una repercusión clara y poderosa en la selección de las conductas de afrontamiento, mientras que las contextuales defienden que son los aspectos más transitorios y ligados a la situación los que determinan la elección. Suelen apuntarse 
los modelos psicodinámicos como prototípicos de las primeras, y el influyente modelo de Lazarus y sus colaboradores de las contextuales (Holahan, Moos y Schaefer, 1996). Desde los psicodinámicos se tiende a hablar de estilos de afrontamiento relativamente estables que traspasan las características específicas de las situaciones y desde el segundo planteamiento se apuntaría hacia la necesidad del microanálisis de la evaluación y afrontamiento de la situación concreta. No obstante, como señalan y defienden Holahan et al. (1996), actualmente se tiende hacia posturas integradoras desde las que se asume que ambas aproximaciones poseen aspectos complementarios para describir el proceso y que tanto los factores personales como los situacionales configuran los esfuerzos de afrontamiento.

Paralelamente, se suele situar la investigación de los mecanismos de defensa como antecedente histórico del estudio del afrontamiento. Mientras que, entre otras razones, se responsabiliza del distanciamiento en los años 70 de lo que podría ser una defensa rasguista de los estilos de afrontamiento (incluso de autores como Folkman o Lazarus) bien a los influyentes postulados situacionistas de autores como Mischel (Costa, Somerfield y McCrae, 1996), o bien al estudio de las respuestas de los sujetos en situaciones altamente estresantes e incluso traumáticas donde se obvian las variables personales ante el protagonismo de la situación (Parker y Endler, 1996). Más adelante, a partir de la década de los 80 , como señalan los últimos autores mencionados, la nueva disposición por el estudio del rasgo se traducirá en un claro interés por las variables del individuo en las investigaciones del afrontamiento.

Por otra parte, si pensamos en el estrés como estímulo ambiental, una de las primeras taxonomías de estresores (Lazarus y Cohen, 1977) dividía los mismos en cambios mayores (que afecten a muchas per- sonas, o que afecten a una o unas pocas) y ajetreos diarios (hassles). Este modo de dividir los acontecimientos en función de su importancia o magnitud «objetiva» ha sido una constante. En esa clasificación que hacían Lazarus y Cohen ya se señalaba que los ajetreos diarios podrían ser extraordinariamente importantes en el proceso de adaptación y conservación de la salud. Otros estudios posteriores han abogado por la misma idea en el sentido de que esas «irritantes, frustrantes, angustiantes demandas que de alguna manera caracterizan la transacción diaria con el ambiente» como los definen Kanner, Coyne, Schaefer y Lazarus (1981, pág. 3) repercuten negativamente sobre la salud tanto física como mental (p. ej., De Longis, Coyne, Dakof, Folkman y Lazarus, 1982; Flanery, 1986a; Flanery 1986b; Johnson y Stone, 1986). De hecho el impacto sobre la salud y el bienestar parece superar al de los acontecimientos vitales más graves e importantes (Holahan Holahan y Belk, 1984; Monroe, 1983; Weinberger, Miner y Tierney, 1987; Wheaton, 1984).

En este estudio, hemos intentado trabajar precisamente con la evaluación y afrontamiento de este tipo de estresores (discusiones con padres, parejas, o amigos, pequeñas frustraciones, falta de tiempo, contratiempos, decepciones, críticas, desengaños.....), para comprobar el papel mediador que sobre tales procesos tienen las variables personales de afectividad, optimismo, perfeccionismo y alexitimia. El haber seleccionado este tipo de variables de personalidad tiene que ver con el hecho de que pensamos que son unas dimensiones que deben ejercer un impacto importante en la evaluación y afrontamiento de las situaciones. La afectividad, al ser entendida como una dimensión que alude al bienestar psicológico subjetivo. El caso del optimismo, por el hecho de que pensamos que las expectativas sobre los resultados, como creencia general, ha de tener su reflejo en los procesos de la 
evaluación y el afrontamiento. El caso del perfeccionismo al compartir la idea de Flett, Hevitt, Blankstein y O'Brian (1991) de que la motivación hacia la perfección ha de ejercer una gran influencia sobre el comportamiento y que esa tendencia a poseer altas metas y criterios en relación con uno mismo, presenta unas características asociadas con una notable vulnerabilidad a los problemas de ajuste. Problemas de ajuste que pensamos pueden provenir del modo de valorar y afrontar las situaciones. La alexitimia por ser un constructo novedoso que pensamos debe de estar relacionado con los procesos de afrontamiento de las situaciones estresantes y sus repercusiones. De manera sintética podríamos señalar que hace referencia a la capacidad disminuida para identificar y/o comunicar sentimientos (Kroner y Forth, 1995) y conceptuarlo como un estilo cognitivo-afectivo que correlaciona positivamente con neuroticismo y psicoticismo (dureza emocional) y negativamente con extraversión (Pandey y Mandal, 1996).

Nos interesaba, en primer lugar, determinar la relación que guardan estas variables con los procesos valorativos y los esfuerzos concretos de afrontamiento; segundo, la capacidad predictiva que sobre las diferentes estrategias de afrontamiento teníamos al contar con valores de todas ellas; y, tercero, la posibilidad de estimar el tamaño del efecto que sobre la evaluación y el afrontamiento (en sus distintas variedades) de este tipo de estresores tenía cada variable por separado y en su conjunto.

\section{MÉTODO}

\section{Sujetos}

Este estudio se ha realizado con la colaboración de estudiantes de psicología. En el mismo han participado 204 sujetos que cumplimentaron alguno de los instrumentos empleados. Todos ellos eran alumnos de segundo curso de Psicología. Sus edades oscilaban en un rango de 27 (mínimo de 21 y máximo de 48 ), con una media de edad $\pm D T=23,42 \pm$ 3,3 años. El $86,4 \%$ eran mujeres y el 14,6 $\%$ hombres. En concreto contamos con 169 estudiantes que respondieron a los cuestionarios que evaluaban las variables de personalidad, y con 188 que cumplimentaron los referentes a la evaluación y el afrontamiento de un ajetreo diario ocurrido recientemente. De todos ellos, 153 constituyen el grupo que contestaron a ambos bloques. De esta manera, cuando a partir de ahora aludamos a resultados que tengan que ver sólo con las variables de personalidad se entenderá que contamos con un $\mathrm{N}=169$; cuando los resultados hagan referencia únicamente a aspectos relacionados con las variables de evaluación y afrontamiento el $\mathrm{N}$ será de 188 y cuando sea preciso tomar en consideración ambos grupos de variables el $\mathrm{N}$ será de 153.

\section{Materiales}

Los instrumentos utilizados han sido autoinformes. A continuación se señalan las variables evaluadas, el nombre resumido con el que se conocerán a partir de aquí, y el instrumento concreto empleado para obtener una medida de las mismas.

1. Variables de Personalidad. Las variables de personalidad evaluadas han sido las de afectividad, optimismo, perfeccionismo y alexitimia.

La Afectividad se evaluó mediante la "Escala de Afectos Positivos y Negativos» (Sánchez-Cánovas, 1994). Este instrumento contiene dos subescalas: Afectividad Positiva y Afectividad Negativa. Cada una de ellas consta de 10 items que 
evalúan los estados de ánimo habituales ofreciendo una puntuación total que es la suma de los estados positivos (alegre, feliz, decidido...) por un lado, y de los negativos (deprimido, furioso, tenso...) por otro, en una escalla de 5 puntos.

El Optimismo, entendido como las expectativas generalizadas sobre resultados positivos frente a negativos, se evaluó mediante el LOT-R (Life Orientation Test) de Scheier, Carver y Bridges (1994). Este instrumento consta de 10 items de los cuales 4 son de relleno. Se responde según una escala de 5 pasos que va desde el total desacuerdo con las afirmaciones, al acuerdo total. Las puntuaciones altas indican alto grado de optimismo.

El Perfeccionismo lo evaluamos mediante el MPS (Multidimensional Perfectionism Scale) de Frost, Marten, Lahart y Rosemblate (1990). Este instrumento ofrece puntuaciones de las 6 dimensiones de perfeccionismo que han hallado los autores. El modo de respuesta consiste en una escala de 5 puntos que van desde "totalmente en desacuerdo» a "total acuerdo». Las puntuaciones altas indican alto grado de perfeccionismo en cada una de sus variantes. Las dimensiones son las siguientes:

- Excesiva preocupación por los errores (en adelante "preocupación errores») que hace referencia a la obsesión por la perfección, por ser el mejor y por no cometer errores, considerando cualquier grado de error como un fracaso.

- Criterios o estándares personales elevados (en adelante «estándares personales») que se refiere a la tendencia a fijarse metas y objetivos superiores a los demás y difíciles, o a veces imposibles, de conseguir.

- Percepción de elevadas expectativas paternas (en adelante "expectativas paternas»), que tiene que ver con el grado en que se perciben a los padres como poseedores de unas expectativas excesivamente altas para con uno mismo.
- Percepción de elevado criticismo paterno (en adelante «criticismo paterno») que alude al grado en que uno piensa que sus padres fueron excesivamente críticos, duros, poco comprensivos y siempre trazando altas metas para uno.

-Dudas sobre la calidad de las propias actuaciones (en adelante "dudas acciones») que tiene que ver con la tendencia a dudar sobre la calidad de la ejecución llevada a cabo por uno mismo y que se asocia con la duda obsesiva y con la insatisfacción con el resultado alcanzado pensando que siempre se puede mejorar.

-Preferencia por el orden y la organización (en adelante «organización»), que es la tendencia a buscar excesivamente el orden, la pulcritud y la organización.

Asimismo se puede obtener una puntuación total en perfeccionismo mediante la suma de las puntuaciones obtenidas en las escalas. En esa suma no se incluyen las puntuaciones obtenidas en estándares personales y en organización. A esta medida en adelante la conoceremos como perfeccionismo total. En algunos casos el nombre de las variables irá precedida de las siglas MPS para agilizar su ubicación.

La Alexitimia la medimos mediante el TAS-20 (Toronto Alexithymia Scale) de Bagby, Taylor y Parker (1992) (traducción de Velasco y Páez, 1996). Esta escala ofrece medidas de los tres factores del constructo que obtuvieron los autores mediante las puntuaciones obtenidas a través de los 20 items que componen el instrumento. El modo de puntuar es el de una escala de 1 a 5 que va desde total desacuerdo al total acuerdo y donde a mayor puntuación mayor es el grado de alexitimia. Los tres factores que incluye la escala son:

-Dificultad para identificar los sentimientos, que alude a la dificultad para identificarlos y distinguirlos de las sen- 
saciones corporales de la emoción (en adelante «identificación sentimientos»).

-Dificultad para describir los sentimientos y para poder expresarlos a los demás (en adelante "descripción de sentimientos»).

-Estilo de pensamiento orientado hacia lo externo, que hace referencia a un estilo cognitivo que es concreto y orientado hacia el mundo externo y no hacia el mundo interno de las emociones, los sentimientos y la subjetividad (en adelante "pensamiento externo»).

Además, podemos obtener una puntuación total en Alexitimia mediante las sumas de las puntuaciones de las escalas (en adelante «alexitimia total»). En algunos casos el nombre de las variables irá precedida de las siglas TAS para facilitar la comprensión.

2. Variables de valoración de la situación.

En cuanto al modo de obtener información sobre la valoración de la situación estresante (el ajetreo diario) se utilizó un cuestionario construido al efecto que contaba de 7 preguntas específicas de la evaluación primaria y secundaria (en términos del modelo de Lazarus). El modo de contestar a cada una de ellas era mediante una escala de 5 puntos de manera que puntuaciones altas estaban indicando que la situación se valoraba en ese sentido. Las preguntas específicas hacían referencia a los aspectos que se relacionan a continuación.

- Importancia, que preguntaba sobre la importancia concedida a la situación, de muy poco a muy importante.

- Malestar, que indagaba el grado de malestar percibido en la situación desde escaso a muy intenso.

-Capacitación, que preguntaba sobre lo capaz que uno se consideraba para resolver la situación y que variaba desde incapaz a totalmente capaz de resolverla.

-Control, referida al grado de control percibido en la situación donde la puntuación iba desde la sensación de incapacidad total para controlar la situación, hasta la certeza de poder controlarla totalmente.

- Cambio, que preguntaba sobre las posibilidades que uno creía tener para cambiar la situación o hacer algo para acabar resolviéndola.

-Desafío, donde se indagaba sobre el grado en que la situación estresante había sido considerada como un desafío ante el que se tenían armas para luchar con efectividad, y que podía variar desde en absoluto a totalmente.

- Amenaza, donde se preguntaba hasta qué punto la situación se había percibido como una amenaza que podría perjudicarle y desbordar sus recursos de afrontamiento.

3. Variables de afrontamiento.

Para obtener medidas de los modos de afrontamiento utilizados se empleó el CSI (Coping Strategy Indicator) de Amirkhan (1990). Este cuestionario consta de tres escalas de 11 items cada una (en una escala de tres puntos donde las puntuaciones altas indican que ese tipo de estrategia se utilizó en gran medida). Las tres dimensiones evaluadas son los tres grandes grupos de estrategias que suelen considerar este tipo de instrumentos. Una primera evalúa las estrategias instrumentales y directas dirigidas hacia la solución del problema que se han utilizado (en adelante «solución de problemas»). Una segunda estaría compuesta por las estrategias de evitación tanto físicas o conductuales como de retirada psicológica - distracción o fantasía- (en adelante «evitación»). La tercera y última evalúa la demanda de apoyo social a través de los demás, bien para conseguir amparo, consuelo o consejo, o la simple búsqueda de contacto humano como necesidad (en adelante «búsqueda de apoyo social»). 


\section{Procedimiento}

El modo de obtener medidas de todas las variables que se acaban de comentar tuvo lugar en dos sesiones distintas. En primer lugar se instó a los sujetos a que cumplimentasen colectivamente los autoinformes que aludían a las variables de personalidad. Una semana después (distanciando ambos tipos de evaluación con el objetivo de que ambas medidas estuviesen tomadas en contextos y situaciones de ánimo que no contaminasen las respuestas a los dos tipos de herramientas en una dirección determinada), se procedió a la cumplimentación de los cuestionarios de evaluación y afrontamiento de un ajetreo diario ocurrido recientemente también de manera colectiva. Para ello se animó a los participantes a que intentasen experimentar de nuevo (rememorándolo) el episodio de la manera más vívida posible y con el mayor número de detalles. Una vez que eso se lograba debían contestar a los cuestionarios. En primer lugar respondían a las preguntas referidas a la valoración primaria y secundaria de la situación y tras ello al cuestionario de afrontamiento, CSI.

\section{RESULTADOS}

\section{Fiabilidad de las pruebas}

Debido a que la mayoría de las pruebas empleadas son de muy reciente utilización en nuestro país y para saber hasta qué punto contábamos con garantías suficientes de que los instrumentos utilizados ofrecían una fiabilidad aceptable, decidimos llevar a cabo los análisis pertinentes, mediante el cálculo del alfa de Cronbach y la correlación dos mitades de Guttman. La práctica totalidad de los resultados fueron moderada o altamente aceptables, en un rango que osciló en el caso del alfa de Cronbach de 0,93 (CSI) a 0,50 (Pensamiento externo, del TAS). Esta última variable y, en menor medida, la escala Criticismo paterno del MPS $(a l f a=0,65)$, fueron las que presentaron los peores índices, ya que en todos los demás casos se superó un valor mínimo de 0,70. Para el estadístico Gutman, el rango de correlaciones osciló entre 0,88 (Evitación, CSI) y 0,47 (pensamiento externo, TAS).

\section{Asociación entre variables}

Como forma de ofrecer una visión preliminar de las relaciones existentes entre las variables de personalidad, evaluación y afrontamiento, decidimos llevar a cabo análisis de correlación Pearson entre pares de variables. En la Tabla 1 pueden apreciarse los valores de las asociaciones que resultaron significativas estadísticamente. En dicha tabla no hemos incluido las relaciones que entre sí mantienen las variables de personalidad puesto que no es el objetivo de interés en este trabajo.

Como se puede apreciar, y destacando lo más llamativo a nivel general, la variable de la valoración de la situación que más relación guarda con las de personalidad es la que hace referencia a lo capaz que uno se cree para solventar la situación estresante (Capacitación). Este aspecto se relaciona negativamente con todas las dimensiones de la alexitimia, con el afecto negativo, varias de las escalas de perfeccionismo y positivamente con el optimismo y el afecto positivo. Las asociaciones de la posibilidad de cambio percibida en la situación (Cambio) guardan cierta similitud con la variable anterior tanto en el sentido como en la magnitud de las mismas. La diferencia estriba en que en este caso las asociaciones con algunas de las escalas del MPS y del TAS no alcanzan la significación estadística. Esa misma simili- 
Tabla 1. Correlaciones Pearson entre las variables de personalidad y las de evaluación y afrontamiento de la situación concreta

\begin{tabular}{|c|c|c|c|c|c|c|c|c|c|c|}
\hline Variables & Import. & Malest. & Capacit. & Control & Cambio & Desafio & Amenaza & $\begin{array}{c}\text { Csi } \\
\text { Solprob. }\end{array}$ & $\begin{array}{c}\text { Csi } \\
\text { Evitac. }\end{array}$ & $\begin{array}{c}\text { Csi } \\
\text { Apoy soc. }\end{array}$ \\
\hline \multicolumn{11}{|l|}{ Importancia } \\
\hline Malestar & $.522^{* *}$ & & & & & & & & & \\
\hline $\begin{array}{l}\text { Capacitación } \\
\text { Control }\end{array}$ & & $\begin{array}{l}-.351^{* *} \\
-336^{* *}\end{array}$ & $586^{* *}$ & & & & & & & \\
\hline $\begin{array}{l}\text { Control } \\
\text { Cambio }\end{array}$ & & $\begin{array}{l}-.35 b \\
.170^{*}\end{array}$ & .586 & $.461^{* *}$ & & & & & & \\
\hline Desafio & $-.164^{*}$ & $-.249^{* *}$ & $.507^{* *}$ & $.512^{* *}$ & $.594^{* *}$ & & & & & \\
\hline $\begin{array}{l}\text { Amenaza } \\
\text { CsiSolProbl }\end{array}$ & $.318^{* *}$ & $.502^{* *}$ & $\begin{aligned}-.428^{* *} \\
294^{* *}\end{aligned}$ & $-.263^{* *}$ & $-.276^{* *}$ & $-.269^{* *}$ & & & & \\
\hline CsiEvitación. & & $.308^{* *}$ & $\begin{array}{l}.294 \\
-.264 * *\end{array}$ & & & & $.297^{* *}$ & & & \\
\hline CsiApoyosoc. & & & & & & & & $.154^{\circ}$ & & \\
\hline Afecto positivo & & & $.166^{*}$ & $.159^{*}$ & & & & & $-.321^{* *}$ & \\
\hline Afecto negativo & & $.179^{*}$ & $-.229^{* *}$ & $-.184^{\star}$ & $-.187^{*}$ & & & $-.197^{*}$ & $.456^{* *}$ & \\
\hline Optimismo & & & $.266^{* *}$ & & $.206^{*}$ & $.208^{*}$ & & $.201^{*}$ & $-.256^{* \star}$ & \\
\hline Preocup. Errores & & & $-.201^{*}$ & & $-.239^{* *}$ & & $.169^{\circ}$ & & $.413^{* *}$ & $-.170^{*}$ \\
\hline \multicolumn{11}{|l|}{$\begin{array}{l}\text { Estánd. Personal. } \\
\text { Expect. Paternas }\end{array}$} \\
\hline Critic. Paterno & & & $-.191^{*}$ & & & & & & $.287^{* *}$ & $-.172^{*}$ \\
\hline Dudas acciones & & & $-.222^{* *}$ & & & & & $-.264^{* *}$ & $.253^{* *}$ & $-.171^{\circ}$ \\
\hline Organización & & & & & & & & $.166^{*}$ & & \\
\hline Perfec. Total & & & $-.227^{* *}$ & & $-.185^{*}$ & $-.186^{*}$ & $.164^{*}$ & $-.215^{* *}$ & $.361^{* *}$ & \\
\hline Identific. Sentim. & & & $-.198^{*}$ & & $-.205^{\circ}$ & $-.186^{\circ}$ & & $-.244^{* *}$ & $.234^{* *}$ & \\
\hline Descripc. Sentim. & & & $-.203^{*}$ & & & & & $-.204^{*}$ & $.274^{* *}$ & $-.299^{* *}$ \\
\hline Pensam. Externo & & & $-.180^{\circ}$ & & & & & $-.217^{* *}$ & & $-.268^{* *}$ \\
\hline Alexitimia total & & & $-.260^{* *}$ & & $-.206^{\circ}$ & $-.167^{\prime \prime}$ & & $-.297^{* *}$ & $.304^{* *}$ & $-.277^{* *}$ \\
\hline
\end{tabular}

Nota: " $p<0.05 " p<0.01$

tud, a nivel global, se mantiene en el caso de la relación entre lo desafiante que se percibió la situación (Desafío) y las variables de personalidad. La diferencia fundamental radica en que en este caso no aparecen asociaciones estadísticamente significativas con la afectividad y, en el caso del perfeccionismo, sólo con la puntuación global.

Por otra parte, la variable referida a lo amenazante que se percibió la situación (Amenaza), tan sólo se relaciona con una medida del perfeccionismo (preocupación por los errores) y con el perfeccionismo como puntuación total, en el sentido de que un mayor grado de perfeccionismo se asocia significativamente con mayor grado de amenaza. El control que uno cree que podría ejercer sobre la situación estresante (Control) tan sólo se asocia con la Afectividad (positivamente con el afecto positivo, y negativamente con el negativo). El malestar percibido en la situación
(Malestar) sólo con el afecto negativo y la importancia concedida a la situación (Importancia) con ninguna de ellas.

Por otra parte sería necesario destacar el hecho de que dos de las escalas de personalidad que hemos empleado no guardan relación significativa con ninguna de las variables de la valoración de la situación ni del afrontamiento de la misma. Nos referimos a "estándares personales» y «expectativas paternas». Y la de "organización» tan sólo aparece relacionada, aunque de manera muy pobre, con la estrategia de solución de problemas.

Por otra parte, si atendemos a las relaciones entre las estrategias del afrontamiento y las variables de personalidad comprobamos que los tres tipos de estrategias se asocian de manera significativa con los cuatro tipos de variables personales. Tan sólo la búsqueda de apoyo social sería independiente de los valores de la 
afectividad y del optimismo. Más en concreto, la evitación se asocia positivamente con las variables «indeseables» tales como la alexitimia, el perfeccionismo (aunque con algunas escalas no) y con el afecto negativo, y negativamente con las «deseables» de optimismo y afecto positivo. Con las estrategias de solución de problemas ocurre lo inverso, salvo en el caso de la variable organización con la que se asocia positivamente. En el caso de la búsqueda de apoyo social lo más llamativo es el hecho de que, como comentábamos más arriba, no se relaciona significativamente ni con el optimismo ni con la afectividad, mientras que sí lo hace negativamente con algunas de las escalas del perfeccionismo y de la alexitimia.

Por último, señalar que de las relaciones que surgen entre evaluación y afrontamiento destaca el hecho de que se establecen asociaciones potentes entre la solución de problemas y capacitación, cambio y desafío en sentido positivo. Entre la evitación y el malestar de manera positiva y la evitación y la capacitación en sentido negativo. Y que la búsqueda de apoyo social no guarda relación significativa alguna con ninguna de las variables de valoración de la situación que hemos considerado.

Como conclusión inicial, señalar que ni la valoración de la situación estresante, ni las estrategias de afrontamiento que se utilizan ante los ajetreos diarios, se muestran independientes de las variables personales que hemos tomado en consideración. Además, cada una de estas dimensiones parece guardar relaciones más estrechas con determinadas variables específicas de la personalidad. Por otro lado constatamos que la solución de problemas y la evitación, como estrategias a emplear, parecen estar fuertemente asociadas a aspectos de la valoración, mientras que la búsqueda de apoyo social como recurso a emplear se muestra independiente de las mismas.
Capacidad predictiva sobre las estrategias de afrontamiento

Una vez que comprobamos que se establecen relaciones significativas y razonables entre las variables de personalidad, evaluación y afrontamiento decidimos comprobar la capacidad predictiva que tendríamos sobre las diferentes estrategias de afrontamiento conociendo los valores de la valoración de la situación estresante que había que afrontar y de las dimensiones de afectividad, optimismo, perfeccionismo y alexitimia.

En el análisis de regresión múltiple (y el stepwise o análisis por pasos) sobre el afrontamiento centrado en el problema como variable dependiente, y tomando como compuesto predictor las variables de valoración y personalidad en su conjunto, encontramos que predecimos el $37,8 \%$ de los intentos operativos de solucionar el problema (el $29 \%$ ajustado). Si llevamos a cabo el análisis stepwise para eliminar variables redundantes o que aporten poco a la predicción, nos encontramos que con tres variables de evaluación (importancia, desafío y cambio), tres dimensiones de perfeccionismo y una de alexitimia (aunque fundamentalmente las de perfeccionismo: estándares personales y dudas sobre las actuaciones) alcanzamos casi el mismo grado de covarianza $(35,5 \%)$. Y si atendemos al índice ajustado alcanzamos un valor superior al anterior $(32,4 \%)$ debido a que contamos con muchas menos variables en el compuesto predictor.

Aunque no incluimos las tablas por cuestiones de espacio, podemos señalar de modo sinóptico que, tomando en cuenta tan sólo las variables de evaluación en la ecuación de regresión comprobamos que predecimos el $23,7 \%$ de la variabilidad del afrontamiento centrado en el problema $\left(R^{2}\right.$ ajustado $\left.=20,7 \%\right)$ y en el stepwise nos quedamos con el $22,6 \%$ $\left(R^{2}\right.$ ajustado $\left.=20,9 \%\right)$ en cuyo modelo de 
regresión aparecerían las variables de importancia, control, desafío y cambio. Si consideramos tan solo las variables que hacen referencia a rasgos de personalidad explicamos el $21,1 \%$ ( $\mathrm{R}^{2}$ ajusta$\mathrm{do}=14,3 \%$ ). En el análisis por pasos el compuesto formado por estándares personales, expectativas paternas, dudas acciones (del perfeccionismo), e identificación sentimientos y pensamiento externo (de alexitimia), explica el $19,3 \%\left(R^{2}\right.$ ajustado=16,6\%).

Llevando a cabo el mismo proceso, pero en este caso situando como variable dependiente la evitación, el análisis de regresión indica que contando con todas las variables de personalidad y de evaluación, predecimos el 39,7\% de los comportamientos de evitación $\left(\mathrm{R}^{2}\right.$ ajustado= $31,1 \%$ ). Si llevamos a cabo el análisis stepwise, nos encontramos que con tres variables de evaluación (capacitación, amenaza y cambio), la afectividad negativa y dos dimensiones de perfeccionismo (preocupación errores y estándares personales) obtenemos un grado de predicción del $34,7 \%\left(\mathrm{R}^{2}\right.$ ajustado= $32 \%$ ).

Como ya hicimos con las estrategias de solución de problemas, sin incluir las tablas por cuestiones de economía de espacio, y llevando a cabo dos nuevos análisis de regresión múltiple (uno con cada tipo de variables -evaluación y personalidad- en el compuesto predictor o explicativo) comprobamos que incluyendo los valores de la valoración del acontecimiento en la ecuación de regresión predecimos el $16 \%$ de la evitación $\left(\mathrm{R}^{2}\right.$ ajustado $\left.=12,8 \%\right)$ y en el análisis por pasos los valores serían del $15 \%$ y del $13,2 \%$ ajustado con las variables de malestar, capacitación, amenaza y desafío. Al considerar tan solo las variables que hacen referencia a las dimensiones de personalidad explicamos el $30 \%\left(\mathrm{R}^{2}\right.$ ajustado $=24 \%$ ) En el análisis stepwise el compuesto formado por Afectividad negativa, preocupación por los errores y estándares personales (en negativo) explicaría el $28,4 \%\left(R^{2}\right.$ ajustado=26,9\%).

Por lo que respecta al modo de afrontamiento caracterizado por la búsqueda de apoyo social y con el compuesto predictor formado con el conjunto de variables de evaluación y personalidad, se alcanza una $R^{2}=27,4$. El valor ajustado, nuevamente decrece de manera llamativa $(17,1 \%)$ puesto que corrige el hecho de contar con muchas variables (19 en total) como predictoras. Llevando a cabo un análisis stepwise comprobamos que explicaríamos el $20,4 \% \quad\left(\mathrm{R}^{2}\right.$ ajusta$\mathrm{do}=17,2 \%$ ) formando parte de la ecuación del modelo de regresión tan sólo las variables de malestar (única de las variables de evaluación), afecto positivo, las dimensiones de alexitimia descripción de sentimientos y pensamiento externo en sentido negativo y preocupación por los errores (también en negativo) y estándares personales de perfeccionismo

Si, como en los casos anteriores, llevamos a cabo este mismo tipo de análisis pero con las variables de evaluación y de personalidad formando compuestos predictores separados, nos encontramos con lo siguiente. Por un lado, con las variables que aluden a la evaluación del acontecimiento, tan sólo somos capaces de explicar el 5,9\% del criterio (llegando al $2,2 \%$ si tomamos el valor ajustado) y el stepwise nos ofrecería un compuesto formado por amenaza y cambio que explicaría el $3,6 \%\left(\mathrm{R}^{2}\right.$ ajustado $\left.=2,6 \%\right)$. Por otra parte, mediante la inclusión en solitario de las variables de personalidad en la ecuación de regresión, alcanzamos un porcentaje de predicción del 19,7\% $\left(\mathrm{R}^{2}\right.$ ajustado=12\%). Y llevando a cabo el procedimiento de selección por pasos nos quedaríamos con las mismas variables (y en el mismo sentido) de personalidad que en el caso en el que contábamos con todas: afecto positivo, las dimensiones de alexitimia descripción de sentimientos y pensamiento externo en sentido negativo 
y preocupación por los errores (también en negativo) y estándares personales de perfeccionismo. Con ellas alcanzaríamos un coeficiente de determinación que implicaría un $16,2 \%$ de explicación de la varianza del criterio $\left(R^{2}\right.$ ajustado $\left.=13,4 \%\right)$.

Todos estos datos los hemos resumido en la Tabla 2 con el propósito de facilitar una representación mental más clara de los resultados.

Como puede apreciarse de manera sintetizada, la capacidad predictiva de los modos de afrontamiento centrados en el problema es muy similar si contamos con información separada de la evaluación de la situación y de las dimensiones de personalidad (superior al $20 \%$ ). Un porcentaje nada desdeñable que aumenta hasta valores por encima del 35\% si combinamos información de los dos tipos de constructos. En el caso de la predicción de los comportamientos de evitación, claramente las variables de personalidad superan en capacidad predictiva al que proviene del conocimiento sobre la valoración realizada. Nuevamente ambos tipos de valores son aceptables (especialmente y de manera llamativa los referidos a personalidad) y si combinamos ambas informaciones nos acercamos al $40 \%$ de predicción de la varianza en afrontamiento centrado en la evitación. En el caso de la búsqueda de apoyo social, resulta poco predecible a partir de la evaluación de la situación y sí que resulta pronosticable a partir del conocimiento de los valores de afectividad, perfeccionismo y alexitimia. Aunando ambos tipos de variables contamos con un poder de predicción nada desdeñable pero claramente inferior al que podemos realizar en el caso de los otros tipos de afrontamiento.

Efectos sobre la evaluación y el afrontamiento de las variables de personalidad y sobre el afrontamiento por parte de la evaluación.

Para comprobar de manera unitaria el efecto que sobre cada una de las estrategias de afrontamiento por separado y sobre todas ellas en su conjunto tiene el tipo de valoración realizada que habíamos evaluado y que aludía a la evaluación primaria y secundaria, llevamos a cabo análisis que nos indicasen el tamaño del efecto en tal relación. En la Tabla 3 aparecen indicados tales valores y en negrita se han destacado aquellos que resultan estadísticamente significativos.

Como puede verse, capacitación $(9,6 \%)$, cambio $(19,2 \%)$ y desafío $(11,4 \%)$ tienen efectos univariados significativos sobre las estrategias de solución de problemas. Malestar $(9,8 \%)$, capacitación $(8,7 \%)$, control $(6,1 \%)$ y amenaza $(11,4 \%)$ sobre la evitación, y ninguna de ellas sobre la búsqueda de apoyo social. A nivel multivariado, excepto la importancia concedida a la situación, todas las demás variables ejercen efectos multivariados significativos estadísticamente sobre los modos de afrontamiento en su conjunto.

Este mismo proceso lo repetimos con las variables de personalidad (ver Tabla

Tabla 2. Comparación de la capacidad predictiva de las variables de evaluación y de personalidad sobre los tres tipos de estrategias de afrontamiento. Datos en porcentajes

\begin{tabular}{lcccccc}
\hline & \multicolumn{2}{c}{ Solución de problemas } & \multicolumn{2}{c}{ Evitación } & \multicolumn{2}{c}{ Búsqueda apoyo social } \\
& Regresión & Stepwise & Regresión & Stepwise & Regresión & \multicolumn{1}{c}{ Stepwise } \\
\hline Evaluación & $23,7(20,7)$ & $22,6(20,9)$ & $16,0(12,8)$ & $15,0(13,2)$ & $5,9(2,2)$ & $3,6(12,0)$ \\
Personalid. & $21,1(14,3)$ & $19,3(16,6)$ & $30,0(24,0)$ & $28,4(26,9)$ & $19,7(12,0)$ & $16,2(13,4)$ \\
Todas var. & $37,8(29,0)$ & $\mathbf{3 5 , 5 ( 3 2 , 4 )}$ & $\mathbf{3 9 , 7}(31,1)$ & $\mathbf{3 4 , 7}(32,0)$ & $27,4(17,1)$ & $20,4(17,2)$ \\
\hline
\end{tabular}

Nota: Entre paréntesis los valores de la correlación múltiple al cuadrado ajustada. 
Tabla 3. Tamaño de los efectos univariados y multivariados de la evaluación de la situación sobre las diferentes estrategias de afrontamiento

\begin{tabular}{|c|c|c|c|c|}
\hline \multirow[b]{2}{*}{ Variables } & \multicolumn{3}{|c|}{ Tamaños de los efectos univariados } & \multirow[b]{2}{*}{$\begin{array}{l}\text { Contraste multivariado } \\
\text { Lambda de Wilks }\end{array}$} \\
\hline & $\begin{array}{l}\text { Solución de } \\
\text { problemas }\end{array}$ & Evitación & $\begin{array}{c}\text { Búsqueda } \\
\text { de apoyo social }\end{array}$ & \\
\hline Importancia & 0.038 & 0.015 & 0.009 & 0.939 \\
\hline Malestar & 0.016 & $0.098^{* *}$ & 0.024 & $0.863^{* *}$ \\
\hline Capacitación & $0.096^{* *}$ & $0.087^{*}$ & 0.045 & $0.796^{* \cdots}$ \\
\hline Control & 0.043 & $0.061^{\circ}$ & 0.011 & $0.892^{\circ}$ \\
\hline Cambio & $0.192^{\cdots}$ & 0.021 & 0.039 & $0.770^{* *}$ \\
\hline Desafío & $0.114^{* * *}$ & 0.006 & 0.036 & $0.831^{* *}$ \\
\hline Amenaza & 0.024 & $0.114^{\ldots * *}$ & 0.020 & $0.849^{* *}$ \\
\hline
\end{tabular}

Nota: " $p<0.05 " p<0.01 \cdots p<0.001$.

4), para lo cual formamos tres grupos de sujetos en cada variable que provenían de la división de los mismos tomando el referente de las puntuaciones que equivalían a los centiles 33 y 66 . Con ello formamos un grupo de bajos, otro de medios y otro de altos en cada una de las variables. Comprobamos que, en orden decreciente y con valores que rara vez llegan al $10 \%$, la identificación de sentimientos, alexitimia total, dudas sobre las acciones, descripción de sentimientos, optimismo y criticismo paterno, tienen efectos significativos sobre la estrategia de afrontamiento que conoce- mos como solución de problemas. Todas las variables, excepto estándares personales, organización (perfeccionismo) y pensamiento externo (alexitimia), tienen efectos significativos (y en general más elevados) sobre la utilización de la evitación. Y sólo dos dimensiones del perfeccionismo (criticismo paterno y dudas sobre las acciones) y tres de las medidas de la alexitimia: estilo de pensamiento externo, alexitimia total y especialmente la capacidad para describir los propios sentimientos, son las variables que tienen efectos significativos sobre la búsqueda de apoyo social.

Tabla 4. Tamaño de los efectos univariados y multivariados de los rasgos de personalidad sobre las diferentes estrategias de afrontamiento

\begin{tabular}{|c|c|c|c|c|}
\hline \multirow[b]{2}{*}{ Variables } & \multicolumn{3}{|c|}{ Tamaños de los efectos univariados } & \multirow[b]{2}{*}{$\begin{array}{c}\text { Contraste multivariado } \\
\text { Lambda de Wilks }\end{array}$} \\
\hline & $\begin{array}{l}\text { Solución de } \\
\text { problemas }\end{array}$ & Evitación & $\begin{array}{c}\text { Búsqueda } \\
\text { de apoyo social }\end{array}$ & \\
\hline Afecto positivo & 0.013 & $0.049^{* *}$ & 0.000 & 0.974 \\
\hline Afecto negativo & 0.030 & $0.123^{\cdots *}$ & 0.004 & $0.857^{\cdots *}$ \\
\hline Optimismo & $0.044^{*}$ & $0.061^{* *}$ & 0.009 & $0.897^{*}$ \\
\hline Preocupación por los errores & 0.015 & $0.167^{* * *}$ & 0.034 & $0.805^{\cdots}$ \\
\hline Eständares personales & 0.017 & 0.012 & 0.013 & 0.960 \\
\hline Expectativas paternas & 0.033 & $0.048^{*}$ & 0.016 & $0.906^{*}$ \\
\hline Criticismo paterno & $0.039^{*}$ & $0.051^{*}$ & $0.054^{*}$ & $0.880^{* *}$ \\
\hline Dudas sobre acciones & $0.087^{\cdots *}$ & $0.049^{*}$ & $0.040^{*}$ & $0.857^{*}$ \\
\hline Organización & 0.028 & 0.013 & 0.010 & 0.958 \\
\hline Perfeccionismo total & 0.038 & $0.120^{* * *}$ & 0.036 & $0.832^{\ldots *}$ \\
\hline Identificación de sentimientos & $0.113^{* * *}$ & $0.100^{* *}$ & 0.021 & $0.803^{* *}$ \\
\hline Descripción de sentimientos & $0.064^{* *}$ & $0.103^{* * *}$ & $0.083^{n *}$ & $0.787^{\cdots *}$ \\
\hline Estilo pensamiento externo & 0.026 & 0.008 & $0.062 *$ & 0.914 \\
\hline Alexitimia total & $0.098^{* *}$ & $0.047^{*}$ & $0.081^{* *}$ & $0.814^{* * *}$ \\
\hline
\end{tabular}

Nota: $" p<0.05 " p<0.01{ }^{* *} p<0.001$ 
A nivel multivariado, únicamente las variables de afecto positivo, estándares personales, organización y pensamiento externo, no tienen efectos multivariados sobre el afrontamiento en su conjunto.

Un último paso era comprobar si, como creíamos, algunas de las variables de personalidad ejercían efectos significativos sobre la valoración de la situación estresante. Antecedente, según la teoría cognitiva, del afrontamiento. Como puede apreciarse (Tabla 5) tan sólo el optimismo ejerce efectos multivariados significativos sobre la evaluación de las situaciones estresantes en su conjunto.

Por lo que respecta a los efectos univariados, vemos que cuando aparecen efectos estadísticamente significativos los valores no son especialmente altos y oscilan entre el 4 y el $7 \%$. La variable sobre la que más constructos de personalidad tienen efectos es la que se refiere a lo capaz que uno se consideraba para resolver la situación (capacitación). Sobre ella tienen efectos: el optimismo, el afecto negativo, dudas sobre acciones y perfeccionismo total y todas las variables de alexitimia. Nuevamente el opti- mismo, junto con las dudas sobre las acciones y dos de las variables de alexitimia (la puntuación total e identificación de sentimientos) tienen efectos significativos sobre las posibilidades que uno creía tener para hacer algo para modificar la situación. Sobre la percepción de la situación como un desafío (relacionada con las dos anteriores), tienen efectos las dudas sobre acciones y la identificación de sentimientos. De esta manera parece que, sintetizando resultados, sobre la sensación de tener algo que hacer en la situación y no ser un mero espectador de la misma que se resigna a su suerte, tiene efectos positivos el optimismo, y negativos las dudas sobre las acciones (perfeccionismo) y la alexitimia (principalmente la dificultad en la identificación de sentimientos). Sobre el resto de variables, como se ve, los efectos son exclusivos de una de las variables. Así, sobre el malestar percibido el criticismo paterno; sobre el control percibido la preocupación por los errores; sobre la sensación de amenaza el perfeccionismo como medida global; y sobre la importancia concedida al acontecimiento ninguna de ellas.

Tabla 5. Tamaño de los efectos univariados y multivariados de los rasgos de personalidad sobre las diferentes variables de evaluación de la situación

\begin{tabular}{|c|c|c|c|c|c|c|c|c|}
\hline \multirow[b]{2}{*}{ Variables } & \multicolumn{7}{|c|}{ Tamaños de los efectos univariados } & \multirow{2}{*}{$\begin{array}{c}\text { Contraste } \\
\text { - multivariado } \\
\text { Lambda de Wilks }\end{array}$} \\
\hline & Importancia & Malestar & Capacitación & Control & Cambio & Desafío & Amenaza & \\
\hline Afecto positivo & 0.000 & 0.000 & 0.032 & 0.029 & 0.008 & 0.005 & 0.017 & 0.922 \\
\hline Afecto Negativo & 0.003 & 0.016 & $0.039^{*}$ & 0.015 & 0.016 & 0.015 & 0.013 & 0.944 \\
\hline Optimismo & 0.021 & 0.029 & $0.057^{*}$ & 0.009 & $0.047^{*}$ & 0.025 & 0.019 & $0.830^{*}$ \\
\hline Preocupación errores & 0.024 & 0.037 & 0.016 & $0.042^{\star}$ & 0.032 & 0.008 & 0.033 & 0.930 \\
\hline Estándares Person. & 0.001 & 0.002 & 0.003 & 0.003 & 0.05 & 0.006 & 0.001 & 0.942 \\
\hline Expectativas pater. & 0.003 & 0.004 & 0.021 & 0.016 & 0.005 & 0.010 & 0.008 & 0.942 \\
\hline Criticismo paterno & 0.009 & $0.044^{*}$ & 0.027 & 0.004 & 0.028 & 0.004 & 0.003 & 0.866 \\
\hline Dudas acciones & 0.005 & 0.001 & $0.057^{*}$ & 0.005 & $0.038^{*}$ & $0.048^{*}$ & 0.036 & 0.861 \\
\hline Organización & 0.018 & 0.010 & 0.020 & 0.013 & 0.007 & 0.009 & 0.025 & 0.897 \\
\hline Perfeccionismo total & 0.006 & 0.008 & $0.063^{* *}$ & 0.022 & 0.036 & 0.020 & $0.043^{*}$ & 0.857 \\
\hline Identificación sentim. & 0.009 & 0.015 & $0.049^{*}$ & 0.016 & $0.050^{*}$ & $0.050^{*}$ & 0.018 & 0.874 \\
\hline Descripción sentim. & 0.010 & 0.003 & $0.068^{* *}$ & 0.016 & 0.036 & 0.016 & 0.015 & 0.852 \\
\hline Pensamiento externo & 0.005 & 0.010 & $0.039^{*}$ & 0.026 & 0.020 & 0.013 & 0.001 & 0.922 \\
\hline Alexitimia total & 0.001 & 0.003 & $0.076^{* *}$ & 0.016 & $0.051^{*}$ & 0.031 & 0.007 & 0.901 \\
\hline
\end{tabular}

Nota: $" p<0.05^{* *} p<0.01{ }^{* *} p<0.001$. 


\section{DISCUSIÓN Y CONCLUSIONES}

Una de las corrientes investigadoras más poderosas y fructíferas que desde hace algún tiempo opera alrededor del fenómeno del estrés es, sin duda, aquella que intenta descubrir las relaciones existentes entre el proceso del estrés, su evaluación y afrontamiento, y la personalidad. Repasando esos estudios nos encontramos con resultados más o menos generalistas. Por nombrar algunos de los ejemplos más representativos diremos que Houtman (1990) defiende que la diferencia entre rasgos de personalidad, estilos de afrontamiento y conductas específicas de afrontamiento radican tan sólo en la generalidad a la que hacen referencia, y Bolger (1990), contundente, afirma que "el afrontamiento es la personalidad en acción ante el estrés»(pag. 525). Otros autores como McCrae y Costa (1986) estiman que son fundamentalmente el neuroticismo y la extraversión, e incluso la apertura a la experiencia, los que determinan modos preferidos de afrontamiento $\mathrm{y}$ en estudios posteriores (Costa, Somerfield y McCrae, 1996) extienden esa influencia a los cinco grandes factores. Algunos más, como Carver, Scheier y Weintraub (1989) defienden que las tendencias estables o modos preferidos (estilos) de afrontamiento se asocian con otras variables personales menos generales como el optimismo, el locus de control, el patrón de conducta tipo A o la ansiedad rasgo. La hipótesis general que todos ellos plantean es que las variables de personalidad, estables a través de largos periodos de tiempo, afectan al funcionamiento psicológico, con lo que parece razonable pensar que deben igualmente afectar a las conductas de afrontamiento, ya que estas no son sino pensamientos, sentimientos y acciones. Por ello, asumiendo que el afrontamiento no es una especie diferente de conducta, se defiende que los rasgos deben dar cuenta de parte de su estabilidad (Costa et al., 1996). Estas variables personales, o mejor dicho, los polos deseables de esas variables, ejercerán un efecto amortiguador (buffering) sobre la vivencia de los estresores, afectando tanto a la interpretación de los mismos como a las respuestas que se emitan ante ellos (McCrae y Costa, 1986; Soriano y Salabert, 1996).

Por su parte, el modelo mediacional cognitivo de Lazarus, Folkman y sus colaboradores dista mucho menos de lo pueda parecer de estos planteamientos, o de lo que algunos de ellos pretenden dar a entender. Es conocido por todos que consideran el estrés como un proceso y que apuntan la necesidad de circunscribirlo a la interacción particular que se establece entre el sujeto y el ambiente en un momento dado. En ese contexto, es cierto que prefieren no hablar de estilos de afrontamiento, pero también lo es (i.e., Lazarus y Folkman, 1984) que no niegan cierta estabilidad en el afrontamiento y que incluso hablan de dos dimensiones formales de estilo de afrontamiento: el flexible frente al rígido y el complejo frente al simple. $Y$ si bien es cierto también que han defendido que las dimensiones tradicionales de personalidad son poco útiles como predictoras, también lo es que en ese mismo trabajo ya señalaban como recursos personales, que ejercían un papel fundamental en la evaluación y en las estrategias de afrontamiento a emplear, la autoestima, el locus de control, la autoeficacia y las habilidades sociales y de solución de problemas entre otros. Más adelante (p. ej., Lazarus y Folkman, 1987; Lazarus, 1991) mencionan como variables importantes en la determinación de la resistencia o vulnerabilidad psicológica al estrés, ya que influyen en los procesos de evaluación y afrontamiento: la autoestima, el locus de control, la autoeficacia, la firmeza, el sentido de coherencia, el optimismo o el patrón de conducta tipo $\mathrm{A}$, e incluso 
consideran el neuroticismo y la extraversión.

La mayoría de estas variables se han investigado por separado y si bien son muchos los autores que se han interesado por el tema, suele existir consenso en los resultados obtenidos. Conscientes de no poder ofrecer una relación exhaustiva, sí podemos reunir algunos de los ejemplos más actuales y representativos. En cuanto al neuroticismo, que quizá haya sido el rasgo más estudiado, se ha visto asociado (de manera general) con un mayor malestar percibido en las situaciones estresantes (Hooker, Monahan, Shifrin y Hutchinson, 1992), y con formas de afrontamiento inefectivo (Denney y Frisch, 1981; Endler y Parker, 1990; McCrae y Costa, 1986), donde predomina la evitación, las reacciones hostiles, la autocrítica, el pensamiento desiderativo y en general estrategias de afrontamiento centradas en la emoción y el menor uso de estrategias centradas en el problema y de búsqueda de apoyo social. En algunos casos, estudiando la ansiedad rasgo como un componente del neuroticismo (p.ej., Jerusalem y Schwarzer, 1989; Smith, Pope, Rhodewalt y Poulton, 1989). Mientras que, aun con resultados no tan claros, se encuentra que la extraversión se asocia con modos de afrontamiento más afectivos, directos, sociales y centrados en el problema (p. ej., Denney y Frisch, 1981; McCrae y Costa, 1986). Otros estudios, la mayoría de ellos centrándose en dos o más variables, investigan el papel de otras dimensiones personales sobre la evaluación o el afrontamiento de los estresores tales como el locus de control, el optimismo, la firmeza o el patrón de conducta tipo A (p. ej., Anderson, 1996; Boland y Cappeliez, 1997; Chang, 1998; Hart y Hittner, 1995; Krause, 1987; Manning, Williams y Wolfe, 1988; Parker, 1994; Scheier y Carver, 1987; Wiebe, 1991). Integrando estos estudios, se pone de manifiesto que la firmeza (control, cambio, autoeficacia, etc.), el locus de control interno, el optimismo y, de manera negativa, los rasgos patógenos del patrón de conducta tipo $\mathrm{A}$, ejercen un efecto amortiguador sobre las consecuencias negativas del enfrentamiento con estresores influyendo, no sólo en las formas de afrontamiento, sino también la valoración de los eventos, convirtiéndose así en fuerzas salutogénicas. Asimismo, son también numerosos los estudios sobre la mediación de la afectividad en el estrés percibido y que también apuntan hacia las ventajas de la afectividad positiva y las desventajas de la negativa (p.ej., Watson, 1988).

De manera más reciente, se han incorporado las variables perfeccionismo y alexitimia, y tras diversos estudios han sido conceptualizadas como factores específicos de vulnerabilidad. Así, trabajos como los de Flett, Hewitt, Blankstein y O'Brian (1991), Flett, Hewitt y De Rosa (1996), Flett, Hewitt y Dyck (1989), y Hewitt y Flett (1993), contemplan el perfeccionismo como un constructo multidimensional del modelo diátesis-estrés, donde las creencias perfeccionistas sirven como diátesis (predisposición a enfermar) asociándose con una notable vulnerabilidad a los problemas de ajuste y prediciéndolos a través de su interacción con el estrés. Por lo que respecta a la alexitimia, son menos numerosos los estudios realizados debido a que es un constructo relativamente reciente. Este constructo ha sido investigado como factor que exacerba el impacto adverso de los estresores sobre la salud física y mental (p. ej., Deary, Scott y Wilson, 1997; Kohn, Gurevich, Pickering y McDonald, 1994), y muy recientemente se ha comprobado que se asocia positivamente con el uso de estrategias de afrontamiento centradas en la evitación (Parker, Taylor y Bagby, 1988).

En nuestro trabajo hemos partido de las hipótesis que se desprenden de todos 
estos planteamientos. Esto es, que las variables de personalidad, que por definición gozan de cierta estabilidad y dotan de consistencia temporal y transituacional al comportamiento, habrían igualmente de afectar y condicionar las conductas propias del afrontamiento. A esto uníamos los planteamientos ampliamente compartidos del equipo de Lazarus y Folkman de que el afrontamiento depende en gran medida de las peculiaridades de la valoración que se lleven a cabo. Y que tanto en un aspecto como en el otro las repercusiones de rasgos más o menos generales y clásicos de la personalidad tienen un papel importante que jugar.

Compartiendo estos planteamientos, hemos pretendido comprobar la repercusión que sobre las conductas específicas del afrontamiento como proceso, tenían variables de personalidad «no clásicas» con las cuales se está comenzando a investigar de manera creciente. Todo ello circunscrito al ámbito del afrontamiento de los ajetreos diarios que se erige como la fuente primordial de los problemas de ajuste en relación con el estrés.

En cuanto a los resultados, a nivel general, se ha comprobado de manera clara que la afectividad, el optimismo, el perfeccionismo y la alexitimia guardan relación con los procesos de valoración y afrontamiento de las situaciones estresantes. Más aún, esa relación que claramente guardan entre ellos comprobamos que se traduce en efectos de la personalidad sobre la evaluación y los comportamientos de afrontamiento. Asimismo queda corroborada la relación que guardan los aspectos de la evaluación con los del afrontamiento como tal, aspecto ya clásico y puesto de manifiesto en otros estudios (p. ej., Soriano, 1994).

Por otra parte, se verifica que es necesario considerar por separado y de manera individualizada las relaciones o efectos de unas variables sobre otras. Así, comprobamos, por ejemplo, cómo deter- minadas dimensiones del perfeccionismo repercuten sobre unos aspectos concretos de la evaluación y no sobre otros, o tienen efectos significativos sobre algunos modos de afrontamiento y no en otros. Lo mismo que ocurre con los aspectos concretos de la valoración de la situación (capacitación, control, amenaza, etc.) y las estrategias de afrontamiento.

En relación con ello habría que señalar que incluso aquellas variables que a través de los diferentes análisis se han ido manifestando como más independientes o mucho menos relacionadas con el proceso de afrontamiento de los ajetreos diarios, aparecen unidas significativamente con alguna de las variables en un análisis determinado como podría ser aportando capacidad predictiva suficiente en una regresión como para ser incluida en el compuesto predictor de un análisis por pasos. Estas variables, que se podrían considerar como menos importantes a nivel global en la evaluación y afrontamiento de las situaciones estresantes habituales serían fundamentalmente: 1) la tendencia a fijarse metas y objetivos superiores a los demás y difíciles o imposibles conseguir (estándares personales); 2) el grado en que uno piensa que sus padres son, o han sido, excesivamente exigentes a la hora de señalar objetivos y metas para uno mismo (expectativas paternas); 3) la predilección por el orden, la pulcritud y la organización (organización) que son tres de las dimensiones del perfeccionismo; y 4) el estilo de pensamiento orientado hacia lo externo (pensamiento externo) como dimensión de la alexitimia, cuya única relación claramente importante radica en su efecto sobre la utilización de la búsqueda de apoyo social como estrategia de afrontamiento, apareciendo como un "estilo cognitivo» que entorpece o debilita la vía que el apoyo social ofrece como modo de afrontar situaciones. 
Esa misma circunstancia se produce con las variables de la valoración en el caso de la importancia subjetiva concedida al acontecimiento que se ha considerado como estresante (importancia), que también se manifiesta como prácticamente independiente de las demás variables medidas.

Un elemento importante que ha surgido de los análisis practicados lo constituye el hecho de que los modos de afrontamiento centrados en el problema se pueden predecir casi en el mismo grado tanto si tenemos información de la evaluación de la situación, como si disponemos de medidas de las dimensiones de personalidad que hemos considerado (sobre todo de perfeccionismo y alexitimia). Y que tal predicción aumenta considerablemente si combinamos información de los dos tipos de constructos. Por su parte, en la predicción de los comportamientos de evitación, si bien una porción importante de la misma se puede realizar desde el conocimiento de las variables de personalidad, es mucho mayor el impacto que sobre ellas tienen las dimensiones de personalidad. Combinando ambos tipos de información nos podemos acercar al $40 \%$ de predicción de la evitación. En el caso de la búsqueda de apoyo social como estrategia de afrontamiento, resulta poco predecible a partir de la evaluación de la situación y sí que lo es (en un porcentaje importante) a partir del conocimiento parcial de la personalidad del sujeto. Nuevamente el contar con la información de los dos tipos de variables nos hace lograr un nivel de predicción importante, aunque inferior al llevado a cabo sobre los otros modos de afrontamiento.

Dicho de otro modo, quizá más gráfico: la afectividad, el optimismo, el perfeccionismo y la alexitimia son variables de personalidad que ejercen una repercusión muy clara sobre los tres modos principales del comportamiento de afronta- miento del estrés. De ellos, destaca su papel en la determinación del uso de la evitación, siguiéndole en importancia su función en la aparición de estrategias de solución de problemas y por último, aunque no por ello carente de importancia, en el uso del apoyo social. Es especialmente interesante el hecho de que en los tres modos de comportamiento estos rasgos superen a los valores aportados por la evaluación de la situación, siendo llamativo sobre todo en el caso de la evitación y en la búsqueda de apoyo social. Asimismo, destaca el hecho de que en el caso de la búsqueda de apoyo social, la evaluación llevada a cabo apenas repercute en este tipo de comportamiento mientras que a través de estos valores de personalidad nos acercamos a un nivel de explicación del $20 \%$. Dicho de otro modo, la búsqueda del apoyo social depende en gran medida de estas dimensiones de personalidad y apenas de la evaluación. La evitación (más que ninguna de las otras), depende fundamentalmente de esas variables de personalidad y moderadamente de la evaluación. $Y$ las estrategias de solución de problemas dependen a partes prácticamente iguales de ambas.

Sin duda alguna, resultados como estos nos tienen que hacer afirmar que ni el afrontamiento es una conducta unitaria y global, ni es únicamente la evaluación de la situación la que determinará los pasos a dar a continuación. Tan importante, o más si nos atenemos a estos resultados, en la determinación de las acciones que realizamos ante los ajetreos diarios, son las dimensiones poco clásicas de afectividad, optimismo, perfeccionismo y alexitimia. Variables que se han manifestado poderosas en la determinación de comportamientos específicos y que seguro habría que unir a otras con el objetivo de poder explicar más claramente y en mayor grado la conducta. 
Concluimos afirmando que los resultados pueden servir de apoyo claro a quienes defienden que factores relativamente estables de la persona poseen una repercusión clara y poderosa en la selección de las conductas de afrontamiento, bien directamente, bien a través de la valoración que se hace de los acontecimientos. No obstante, también es de rigor reconocer que los «contextualistas» encontrarían respaldo a sus planteamientos al comprobar que una parte importante de la varianza de tales comportamientos queda sin explicar, o que los tamaños de los efectos de cada variable por separado son excesivamente pequeños como para otorgar a esas variables por separado el papel de eje director del comportamiento encubierto o manifiesto ante los ajetreos diarios. Quizá, como en la mayoría de los casos, ambas cosas sean ciertas.

\section{REFERENCIAS BIBLIOGRÁFICAS}

Amirkhan, J. (1990). A factor analytically derived measure of coping. The coping strategy indicator. Journal of Personality and Social Psychology, 59, 1066-1074.

Andersson, G. (1996). The benefits of optimism: A meta-analytic review of life orientation test. Personality and Individual Differences, 21, 719-725.

Bagby, R.M., Taylor, G.J., y Parker, J.D. (1992). Twenty-item Toronto Alexithymia Scale. Item selection and cros-validation of the factor structure. Journal of Psychosomatic Research, 38, 23-32.

Boland, A., y Cappeliez, P. (1997). Optimism and neuroticism as predictors of coping and adaptation in older women. Personality and Individual Differences, 22, 909919.

Bolguer, N. (1990). Coping as a personality process: A prospective study. Journal of personality and social psychology, 59, 525-537.

Carver, C.S., Scheier, M.F., y Weintraub, J.K. (1989). Assesing coping strategies: A theoretically based approach. Journal of Personality and Social Psychology, 56, 267-283.
Chang, E. (1998). Dispositional optimism and primary and secondary appraisal of stressor. Controlling for confounding influences and relations to coping and psychological and physical adjustment. Journal of Personality and Social Psychology, 74, 1109-1120.

Costa, P., Somerfield, M., y McCrae, R. (1996). Personality and coping: a reconceptualization. En M. Zeidner y N. Endler (Eds.), Handbook of coping. Toronto: John Wiley and Sons.

De Longis, A., Coyne, J.C., Dakof, G., Folkman, D., y Lazarus, R.S. (1882). Relationships of daily hassles, uplifts and major life events to health status. Health Psychology, 1, 119-136.

Deary, I.J., Scott, S., y Wilson, J.A. (1997). Neuroticism, alexithymia and medically unexplained symptoms. Personality and Individual Differences, 22, 551-564

Denney D.R., y Frisch, M.B. (1981). The role of neuroticism in relation of stress and illness. Journal of Psychosomatic Research, 25, 303-397

Endler, N.S., y Parker J.D.A. (1990). Multidimensional assessment of coping: A critical evaluation. Journal of Personality and Social Psychology, 58, 844, 854.

Flannery, R.B. (1986a). Major life events and daily hassles in predicting health status: methodological inquiry. Journal of Clinical Psychology, 42, 485-487.

Flannery, R.B. (1986b). Negative affectivity, daily hassles, and somatic illness: preliminary inquiry concerning hassles measurement. Educational and Psychological Measurement, 46, 1001-1004.

Flett, G.L., Hewitt, P.L., Blankstein, K., y O’Brian, S. (1991). Perfectionism and learned resourcesfulness in depresion and selfesteem. Personality and Individual Differences, 12, 61-68.

Flett, G.L., Hewitt, P.L., y De Rosa, T. (1996). Dimensions of perfectionism, psychosocial adjustment and social skills. Personality and Individual Differences, 20, 143-150.

Flett, G.L., Hewitt, P.L., y Dyck, T. (1989). Self-oriented perfectionism, neuroticism and anxiety. Personality and Individual Differences, 10, 731-735.

Frost, R.O., Marten, P., Lahart, C., y Rosemblate, R. (1990). The Dimensions of perfec- 
tionism. Cognitive Therapy and Research, 14, 449-468.

Hart, K.E., y Hittner, J.B. (1995). Optimism and pessimism. Associations to coping and anger-reactivity. Personality and Individual Differences, 19, 827-839.

Hewitt, P.L., y Flett, G.L. (1993). Dimensions of perfectionism, daily stress, and depresion: A test of the specific vulnerability hypothesis. Journal of Abnormal Psychology, 102, 58-65.

Holahan, C.K., Holahan, C.J., y Belk, S.S. (1984). Adjustment in aging: the roles of life stress, hassles and self-efficacy. Health Psychology, 3, 315-328.

Holahan, C., Moos, R., y Schaefer, J. (1996). Coping, stress resistance and growth. En M. Zeidner y N. Endler (Eds.), Handbook of coping. Toronto: John Wiley and Sons.

Hooker, K., Monahan, D., Shifrin, K., y Hutchinson, C. (1992). Mental and physical health of espouse caregivers: The role of personality. Psychology and aging, 7, 367-375.

Houtman, I.L.D. (1990). Personal coping resources and sex differences. Personality and Individual Differences, 11, 53-63.

Jerusalem, M., y Schwarzer, R. (1989). Anxiety and self-concept as antecedents of stress and coping. A longitudinal study with German and Turkish adolescents. Personality and Individual Differences, 10, 785-792.

Johnson, M., y Stone, G. L. (1986). Social workers and bournout: a psychological description. Journal of Social Service Research, 3, 315-328.

Kanner, A.D., Coyne, J.C., Schaefer, C., y Lazarus, R.S. (1981). Comparisons of two modes of stress measurement: daily hassles and uplifts versus major life events. Journal of Behavioral Medicine, 4, 1-39.

Kohn, P.M., Gurevich, M., Pickering, D.I., y McDonald, J.E. (1994). Alexithymia, reactivity and the adverse impact of hasslesbased stress. Personality and Individual Differences, 16, 805-812.

Krause, N. (1987). Chonic strain, locus of control, and distress in adults. Psychology and Aging, 2, 375-382.

Kroner, D.G., y Forth, A.E. (1995). The Toronto Alexithymia Scale with incercerated ofenders. Personality and Individual Differences, $19,625-634$.
Lazarus, R.S. (1991). Emotion and Adaptation. Nueva York: Oxford University Press.

Lazarus, R.S., y Cohen, J.B. (1977). Environmental stress. En I. Altman y J. F. Wohlwill (Eds.), Human behavior and the environment: Current theory and research. Nueva York: Plenum Press.

Lazarus, R.S., y Folkman, S. (1984). Stress Appraisal and Coping. Nueva York: Springer Publishing Company, Inc.

Lazarus, R.S., y Folkman, S. (1987). Transactional theory and research on emotions and coping. European Journal of Personality, 1, 141-169.

Manning, M.R., Williams R.F., y Wolfe, D.M. (1988). Hardiness and the relationship between stressors and outcomes. Work and Stress, 2, 205-216.

McCrae, R., y Costa, P.T. (1986). Personality, coping, and coping effectiveness in adult sample. Journal of Personality, 54, 385-405.

Monroe, S.M. (1983). Major and minor life events as predictors of psychological distress: further issues and findings. Journal of Behavioral Medicine, 6, 189-205.

Pandey, R., y Mandal, M.K. (1996). Eysenckian personality dimensions and alexithymia: Examing the overlap in terms of perceived autonomic aurousal. Personality and Individual Differences, 20, 499-504.

Parker, J.D.A., Taylor, G.J., y Bagby, R.M. (1998). Alexithymia: Relationship with ego defense and coping styles. Comprehensive Psychiatry, 39, 91-98.

Parker, J., y Endler, N. (1996). Coping and defence. A historical overview. En M. Zeidner y N. Endler (Eds.), Handbook of coping. Toronto: John Wiley and Sons.

Parker, K.R. (1994). Personality and coping as moderators of work stress procesess: Models, methods and measures. Special Issues: A healthier work environment. Work and Stress, 8, 110, 129.

Sánchez-Cánovas, J. (Dir.). (1994). Relaciones entre salud física, psíquica y estrés, estilos de vida slaudables y apoyo social: un estudio exploratorio en mujeres climatéricas. Investigación subvencionada por el Fondo de Investigaciones Sanitarias de la Seguridad Social. Ministerio de Sanidad y Consumo. Expediente: 94/0565. Manuscrito no publicado. 
Scheier, M.F., y Carver, C.S. (1987). Dispositionaloptimism and physical well-being: The influence of generalized outcome expectancies on health. Special Issue. Personality and Physical health. 55, 169-210.

Scheier, M.F., Carver, C.S., y Bridges, M.W. (1994). Distinguishing Optimism from Neuroticism (and trait ansiety, self-mastery, and self-esteem): A reevaluation of the Life Orientation Test. Journal of Personality and Social Psychology, 67, 10631078.

Smith, T.W., Pope, M.K., Rhodewalt F., y Poulton, J.L. (1989). Optimism, Neuroticism, Coping, and symptom reports: And alternative interpretation of the Life Orientation Test. Journal of Personality and Social Psychology, 56, 640-648.

Soriano, J. (1994). Las estrategias de afrontamiento desde una perspectiva transaccional. Boletín de Psicología, 44, 77-92.

Soriano, J., y Salavert, L. (1996). The hospital anxiety and depression scale questionaire in physical anger and evaluation situa- tions. Counselling Psychology Quartery, 9, 281-296.

Velasco, C., y Páez, D. (1996). Alexitimia, comunicación emocional y problemas psicosomáticos. Boletín de Psicología, 52, 25-46.

Watson, D. (1988). Individual and interindividual analyses of positive and negative affect: Their relation to health complaints, perceived stress, and daily activities. Journal of Personality and Social Psychology, 54, 1020-1030.

Weinberger, M., Miner, S.L., y Tierney, W.M. (1987). In support of hassles measure of stress in predicting health outcomes. Journal of Behavioral Medicine, 10, 19-31.

Wheaton, B. (1994). Samplig the stress universe. En W. R. Avison y I. H. Gottig (Eds.), Stress and mental health. Contemporary issues and prospects for future. Nueva York: Plenum Press.

Wiebe, D.J. (1991). Hardiness and stress moderation. A test of proposed mechanism. Journal of Personality and Social Psychology, 60, 89-99. 